\title{
Case Report: Spontaneous Resorption of Spinal Disk Herniation and Its Adjacent-level Complications: ACase Report
}

\author{
Kseniya Yurku ${ }^{1,2}$ (D), Sergey Bondarev ${ }^{1}$ (D), Irina Lazareva ${ }^{1}$ (D), Tatiana Krasavina ${ }^{1}$ (D), Mark Ivanov ID $^{3}$ \\ 1. Departmentof Sports Medicine and Medical Rehabilitation, I.M. Sechenov First Moscow State Medical University (Sechenov University), Moscow, Russia. \\ 2. Center of Sports Rehabilitation, ReMedica, Moscow, Russia. \\ 3. Khimki Regional Hospital, Moscow Region, Moscow, Russia.
}

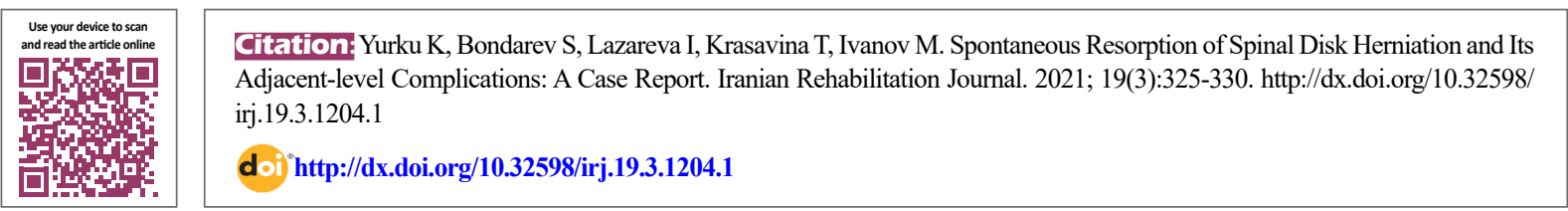

\section{(c) (1) (8)}

\section{Article info:}

Received: 11 Aug 2020

Accepted: 02 Feb 2021

Available Online: 01 Sep 2021

\section{Keywords:}

Spinal disk herniation, Low back pain, Spontaneous resorption

\section{ABSTRACT}

Objectives: Symptoms associated with spinal disk herniation may heal without surgery. In some patients, imaging reveals a reduction in hernia size, too. This phenomenon is referred to as spontaneous regression of the herniated disk. It was first reported in 1984 and is widely covered in today's research papers.

Case Presentation: This paper describes a case of spontaneous regression of lumbar disk herniation at L5-S1, where another disk was herniated at L4-L5 after only 8 months. The patient (F) sought help complaining of pain in the lumbar region. An MRI scan revealed a 6.2-mm hernia at L5-S1. The patient was prescribed conservative treatment comprising non-steroidal anti-inflammatory drugs and kinesitherapy, to which she refused to adhere and decided to treat the condition on her own. Eight months later, the patient sought help again, reporting other symptoms of pronounced constant pain in the rear lateral surface of the left leg and lameness. A further MRI scan revealed spontaneous hernia resorption at L5-S1 with an emergent vacuum phenomenon and a different disk herniated at L4-L5 to $9.4 \mathrm{~mm}$.

Discussion: Since the symptoms were pronounced and non-steroidal anti-inflammatory drugs did not have a therapeutic effect, the patient required surgery.

\section{* Corresponding Author:}

Kseniya Yurku, MD.

Address: Departmentof Sports Medicine and Medical Rehabilitation, I.M. Sechenov First Moscow State Medical University (Sechenov University), Moscow, Russia.

Tel: +7 (926) 6487864

E-mail: kpredatko@mail.ru 


\section{Highlights}

- Spontaneous regression of the herniated disk requires more research, as its underlying causes are not entirely known.

- Rapid regression of the hernia, possibly, can provoke degenerative changes in the adjacent regions of the spine.

- The patient with spontaneous or induced hernia resorption should be followed up for a long time and continuous MRI scanning to prevent degeneration of intervertebral disks in adjacent segments.

\section{Plain Language Summary}

Resorption of a herniated disk is a condition when the hernia, on its own or during treatment, decreases in size. This phenomenon was first reported in 1984 and is widely covered in today's research papers. Some researchers suggest such a case is the best treatment outcome. In our case, after spontaneous resorption of the hernia, another hernia appeared in the adjacent region of the spine, which required surgical intervention. We believe that patients who have experienced rapid spontaneous or induced resorption should be under the supervision of specialists for an extended period, despite regression of pain and improvement in general physical condition.

\section{Introduction}

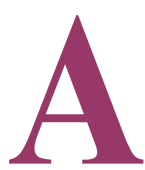

herniated disk (or disk herniation) is one of the most frequent causes of low back pain and radicular syndrome, even in young people $[1,2]$. Today, intervertebral disk degeneration can be treated both conservatively and surgically [3]. The choice of treatment depends not only on the doctor but also on the patient. Symptoms associated with a herniation may heal without surgery; in some patients, imaging reveals a reduction in hernia size [4]. This phenomenon is referred to as spontaneous regression of the herniated disk, which may be partial or complete.

This phenomenon has been known for a long time. Intervertebral herniation resorption on top of conservative treatment was first reported in $1984[5,6]$. The last decade has seen numerous reports of spontaneous and induced herniated disk regression $[7,8]$.

Animal studies prove that annulus fibrosus can heal after experimentally induced damage [9]. As of today, there is no rigorous evidence that this event could happen in the human body as well; however, granulation tissue patterns obtained during surgery indicate that it might take place in humans, too [10]. This is why intervertebral herniated disk resorption is mainly considered a sign of positive process dynamics in today's papers, but there is little information on how the adjacent spine segments respond to such regression [11].
Below is the case of a patient that had spontaneous regression of the herniated disk at L5-S1 with an emergent vacuum phenomenon that resulted in a herniation at a higher level, requiring further surgery.

\section{Case Presentation}

Our case was a 29-year-old female who sought help for the low back pain for seven days. She had no history of irradiation. This condition had been periodically observed after pregnancy for two preceding years. She sought no help, as pain regressed in two to three days without intervention. The patient thought her condition could be a result of lifting her child with arms too quickly. She said she had not had any injuries, chronic diseases, or surgeries. Her Visual Analog Scale (VAS) pain score was 4 . Neurological tests did not identify any abnormalities. The patient was MRI-scanned, which revealed a 2-mm circular dorsal protrusion of the L4-L5 disk and 6.2-mm median/paramedian herniation of the L5-S1 disk (Figure 1).

Due to a lack of indications for surgery, the patient was prescribed Non-Steroidal Anti-Inflammatory Drugs (NSAIDs) and kinesitherapy. She refused to follow the treatment plan and decided to treat the condition herself.

Eight months later, the patient sought help again, this time with more severe complaints. She reported persistent aching pain in the buttock and the rear lateral surface of the left leg. The pain got worse at rest. The left leg was asthenic, could not be lifted, and her toe was numb. The pain emerged gradually over three days and became unbear- 

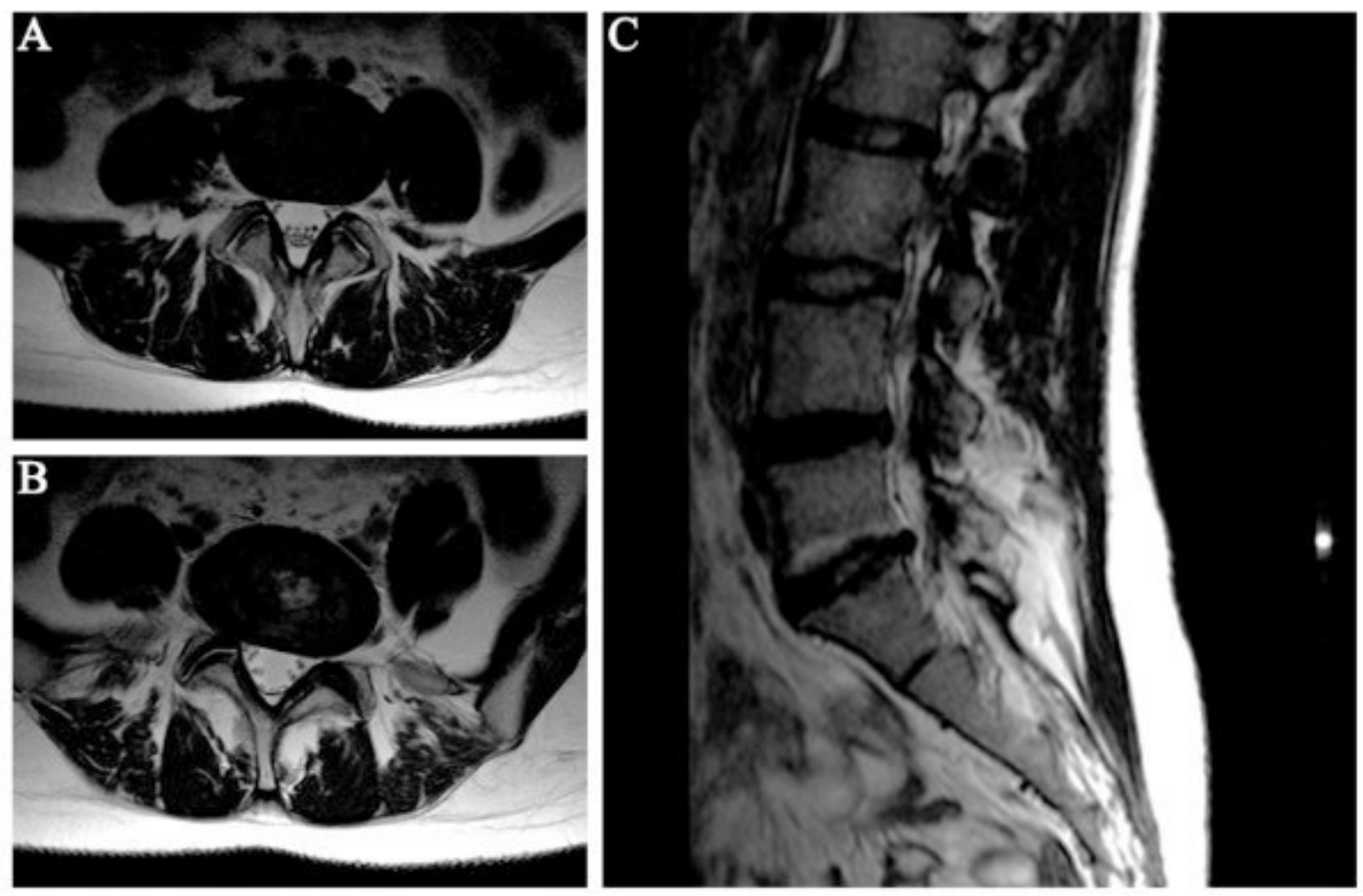

Figure 1. Lumbar MRI scan, first treatment

Iranian Rehabilitation \ournal

A: L4-L5 Axial Plane; B: L5-S1 Axial Plane; C: Sagittal plane: L4-L5 disk protrusion, L5-S1 disk herniation.

able. She tried NSAID-based self-treatment, but it did not have even the slightest analgesic effect. The patient said she had been physically active for the past eight months, exercised a lot, and had no recurrence of back pain.

The VAS score was eight this time. Vertebral flexion, extension, and lateral flexion aggravated leg pain. The Lasègue sign was positive at $10^{\circ}$ left-side with acute pain in the buttock and the rear lateral surface of the leg down to the ankle; the pain aggravated over time.

The patient was MRI-scanned again, which revealed a 9.4-mm paramedian dorsal herniation of the L4-L5 disk and a 2.8-mm median/paramedian herniation of the L5S1 disk (Figure 2).

Based on the MRI data, a neurological examination, and the fact that NSAIDs were not effective, the patient was undergone surgery: microdiscectomy L4-L5. The VAS score dropped to 1-2 on day 2 after surgery.

\section{Discussion}

Most patients that experience disk-related back pain are known to recover without surgery. 12 Still, it has long been believed that full recovery means the disappearance of clinical symptoms, while morphological changes in the spine were not taken into account as a recovery cri- terion. In recent decades, researchers have reported that a herniated disk can be resorbed spontaneously. Still, there is little evidence of how adjacent spine segments react to the process [12].

Hakan and Gurcan11 presented a patient with L5-S1 disk extrusion three years after L4-L5 disk herniation was resorbed. This event can be seen as a downward reaction of the intervertebral disk, which, judging from the spine structure, should be a more frequent case. The case discussed in this paper is, on the other hand, that of an upward reaction. Moreover, the two MRI scans were separated by eight months, a sign that resorption was fast, and so was the new herniation.

The regression rate seems to depend on how pronounced the inflammatory process is. As the annulus fibrosus breaks and the nucleus pulposus is released, it triggers an inflammatory response associated with many inflammatory neurotransmitters produced from the nucleus pulposus cells. These transmitters stimulate the accumulation of cellular infiltration, mainly by macrophages, which facilitates a higher expression of the factors that cause the extracellular matrix to degrade, thus resulting in herniated disk resorption. In other words, the higher the neurotransmitter production rate, the faster the intervertebral herniated disk will be resorbed. 

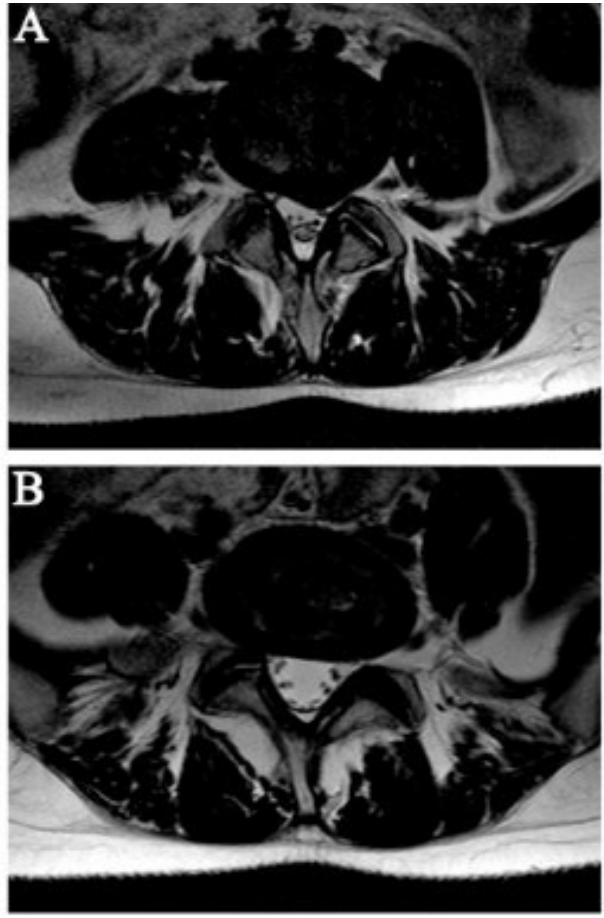

Figure 2. Lumbar MRI scan eight months later

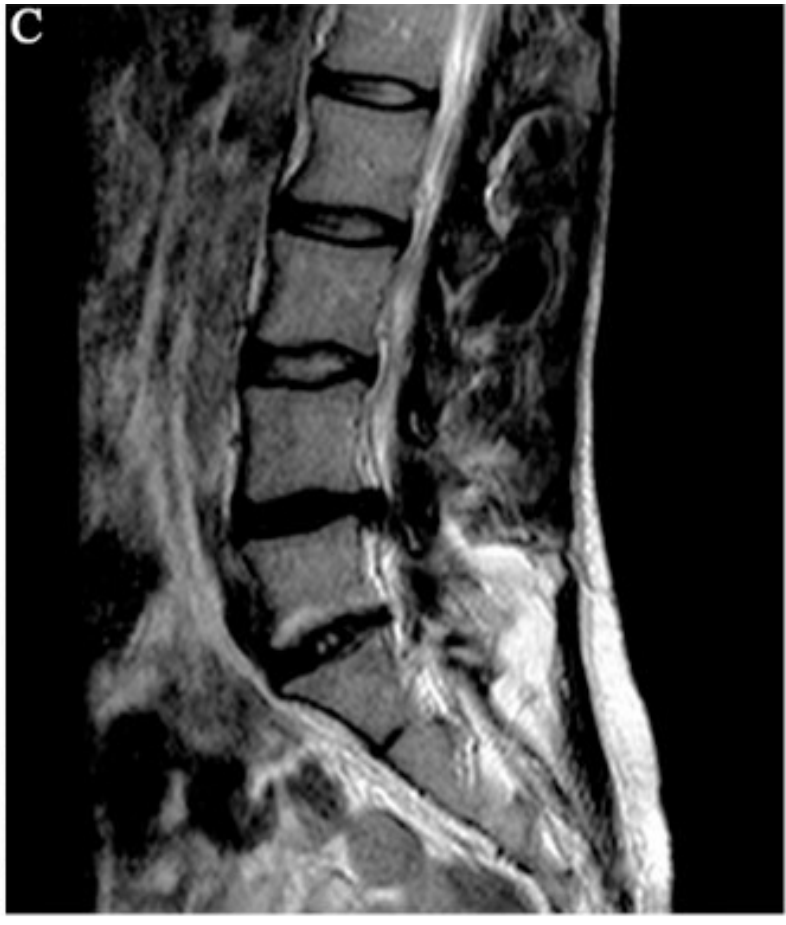

Iranian Rehabilitation \ournal

A: L4-L5 axial plane; B: L5-S1 axial plane; C: Sagittal plane: Herniated L4-L5 disk, L5-S1 disk partially resorbed.

At the same time, from the standpoint of physiology and biomechanics, rapid change in the spine structure like fast hernia resorption may induce a compensatory reaction above or below, as the adjacent disks take over the cushioning load. This outcome was seen in our patient, as her L5$\mathrm{S} 1$ herniation was reduced from $6.2 \mathrm{~mm}$ to $2.8 \mathrm{~mm}$, but the associated vacuum phenomenon caused a 9.4-mm L4-L5 disk herniation. Whether spontaneous or induced, the resorption process requires long-term monitoring and followup to prevent complications in adjacent spine segments.

\section{Conclusion}

The causes of spontaneous regression of a herniated disk have remained unclear. Some researchers suggest such resorption is the best treatment outcome. To date, there is little to no data on the negative consequences of this process. However, this case shows that the regression of one intervertebral disk may cause adverse effects in adjacent segments in the long term. Thus, any patient with spontaneous or induced hernia resorption should be subject to long-term follow-up and continuous MRI scanning to prevent degeneration of intervertebral disks in adjacent segments.

\section{Ethical Considerations}

\section{Compliance with ethical guidelines}

All ethical principles are considered in this article. The participant was informed of the purpose of the case report and its implementation stages. She was also assured about the confidentiality of her information and was free to leave the study whenever she wished, and if desired, the research results would be available to them. A written consent has been obtained from the subject. Principles of the Helsinki Convention was also observed.

\section{Funding}

This research did not receive any grant from funding agencies in the public, commercial, or non-profit sectors.

\section{Authors' contributions}

All authors equally contributed to preparing this article.

\section{Conflict of interest}

The authors declared no conflict of interest. 


\section{Acknowledgments}

We express our gratitude to the «ReMedica» Sports Rehabilitation Center for the information provided about the patient.

\section{References}

[1] Karademir M, Eser O, Karavelioglu E. Adolescent lumbar disc herniation: Impact, diagnosis, and treatment. Journal of Back and Musculoskeletal Rehabilitation. 2017; 30(2):347-52. [DOI:10.3233/BMR-160572] [PMID]

[2] Peng B, Hao J, Hou S, Wu W, Jiang D, Fu X, et al. Possible pathogenesis of painful intervertebral disc degeneration. Spine 2006; 31(5):560-6. [DOI:10.1097/01.brs.0000201324.45537.46] [PMID]

[3] Rahmathulla G, Kamian K. Lumbar disc herniations 'to operate or not' patient selection and timing of surgery. Korean Journal of Spine 2014; 11(4):255-7. [DOI:10.14245/ kjs.2014.11.4.255] [PMID] [PMCID]

[4] Kim ES, Oladunjoye AO, Li JA, Kim KD. Spontaneous regression of herniated lumbar discs. Journal of Clinical Neuroscience. 2014; 21(6):909-13. [DOI:10.1016/j.jocn.2013.10.008]

[5] Guinto FC, Hashim H, Stumer M. CT demonstration of disk regression after conservative therapy. American Journal of Neuroradiology. 1984; 5(5):632-3. [PMID][PMCID]

[6] Teplick JG, Haskin ME. Spontaneous regression of herniated nucleous pulposus. American Journal of Roentgenology. 1985; 145(2):371-5. [DOI:10.2214/ajr.145.2.371] [PMID]

[7] Kim SG, Yang JC, Kim TW, Park KH. Spontaneous regression of extruded lumbar disc herniation: Three cases report. Korean Journal of Spine. 2013; 10(2):78-81. [DOI:10.14245/ kjs.2013.10.2.78] [PMID] [PMCID]

[8] Kesikburun B, Eksioglu E, Turan A, Adiguzel E, Kesikburun $\mathrm{S}$, Cakci A. Spontaneous regression of extruded lumbar disc herniation: Correlation with clinical outcome. Pakistan Journal of Medical Sciences. 2019; 35(4):974-80. [DOI:10.12669/ pjms.35.4.346] [PMID] [PMCID]

[9] Inoue G, Ohtori S, Aoki Y, Ozawa T, Doya H, Saito, T, et al. Exposure of the nucleus pulposus to the outside of the anulus fibrosus induces nerve injury and regeneration of the afferent fibers innervating the lumbar intervertebral discs in rats. Spine. 2006; 31(13):1433-8. [DOI:10.1097/01. brs.0000219946.25103.db] [PMID]

[10] Peng B, Wu W, Hou S, Li P, Zhang C, Yang Y. The pathogenesis of discogenic low back pain. The Journal of Bone and Joint Surgery. 2005; 87(1):62-7.[DOI:10.1302/0301620X.87B1.15708]

[11] Hakan T, Gurcan S. Spontaneous regression of herniated lumbar disc with new disc protrusion in the adjacent level. Case Reports in Orthopedics. 2016; 2016:1538072. [DOI:10.1155/2016/1538072] [PMID] [PMCID]

[12] Ikeda $T$, Nakamura $T$, Kikuchi $T$, Umeda $S$, Senda $H$, Takagi K. Pathomechanism of spontaneous regression of herniated lumbar disc: Histologic and immunohistochemical study. Journal of Spinal Disorders. 1996; 9(2):136-40 [DOI:10.1097/00002517-199604000-00009][PMID] 
This Page Intentionally Left Blank 